Journal of

Synchrotron

Radiation

ISSN 0909-0495

Received 27 February 2012 Accepted 5 December 2012

\section{Sagittal focusing of synchrotron radiation X-rays using a winged crystal}

\author{
A. Nisawa, ${ }^{\text {a* }}$ Y. Yoneda, ${ }^{\text {b }}$ G. Ueno, ${ }^{\text {a }}$ H. Murakami, ${ }^{a}$ Y. Okajima, ${ }^{b}$ K. Yamamoto, \\ Y. Senba, ${ }^{c}$ K. Uesugi, ${ }^{c}$ Y. Tanaka, ${ }^{a}$ M. Yamamoto, ${ }^{a}$ S. Goto ${ }^{c}$ and T. Ishikawa ${ }^{a, c}$ \\ ${ }^{a}$ RIKEN Harima Institute SPring-8 Center, Kouto 1-1-1, Sayo, Hyogo 679-5148, Japan, ${ }^{\mathbf{b}}$ Kansai \\ Photon Science Institute, Japan Atomic Energy Agency (JAEA), Kouto 1-1-1, Sayo, Hyogo 679-5148, \\ Japan, and 'Japan Synchrotron Radiation Research Institute (JASRI), Kouto 1-1-1, Sayo, Hyogo \\ 679-5198, Japan. E-mail: nisawa@spring8.or.jp
}

\begin{abstract}
A Si(111) winged crystal has been designed to minimize anticlastic bending and improve sagittal focusing efficiency. The crystal was thin with wide stiffening wings. The length-to-width ratio of the crystal was optimized by finite element analysis, and the optimal value was larger than the 'golden value'. The analysis showed that the slope error owing to anticlastic bending is less than the Darwin width. The X-rays were focused two-dimensionally using the crystal and a tangentially bent mirror. The observed profiles of the focal spot agreed well with the results of a ray-tracing calculation in the energy range from 8 to $17.5 \mathrm{keV}$. $\mathrm{X}$-ray diffraction measurements with a high signal-to-noise ratio using this focusing system were demonstrated for a small protein crystal.
\end{abstract}

Keywords: sagittal focusing; double-crystal monochromator; winged crystal; two-dimensional focusing; crystal bender; SPring-8.

\section{Introduction}

Two-dimensional focusing by combining a bent second crystal in a double-crystal monochromator (Sparks et al., 1980, 1982) and a tangential-focusing mirror is an efficient method of increasing the flux density at bending-magnet and wiggler beamlines (e.g. Borsboom et al., 1998; Bilsborrow et al., 2006; Koshelev et al., 2009; Nomura \& Koyama, 1999; Yoneda et al., 2005). Another efficient method is the combination of a double-crystal monochromator with plane-plane crystals and a two-dimensional focusing mirror (Kirkpatrick-Baez mirror, toroidal mirror or tangentially bent cylindrical mirror). The advantages of combining a sagittally bent second crystal and a tangential-focusing mirror over two-dimensional focusing mirror optics are (i) a fixed-height exit over a wide energy range, (ii) a higher flux gain owing to the wider horizontal acceptance, and (iii) the capability of high-energy focusing. In this method the beam size in two-dimensional focusing is sensitive to the shape error of the second crystal from the ideal cylindrical shape owing to anticlastic bending. The anticlastic bending of the second crystal (Sparks et al., 1982; Kushnir et al., 1993) leads to a technical difficulty in controlling the sagittal radius of curvature and the parallelism between the first and second crystals within the Darwin width. The anticlastic bending causes undesirable aberrations and the loss of photon flux in sagittal focusing. Various solutions have been proposed to minimize anticlastic bending, such as by using a ribbed (Borsboom et al., 1998; Bilsborrow et al., 2006), hinged or slotted crystal (e.g. Sparks et al., 1982; Kushnir et al., 1993; Schulze et al., 1998; Yoneda et al., 2001; Feng et al., 2008; Koshelev et al., 2009) with various types of bend mechanism for bending-magnet, wiggler and undulator beamlines. Since a slotted crystal has a smoother reflective surface than both ribbed and hinged crystals, it is suitable for fine focusing. The magnitude of anticlastic bending depends on the aspect ratio of a rectangular crystal. The anticlastic bending at the center of a crystal is minimized at the 'golden value' of 1.42 (Kushnir et al., 1993), which is the ratio for a rectangular $\operatorname{Si}(111)$ focusing crystal with 'clamped' or 'built in' boundary conditions (Kushnir et al., 1993; Quintana et al., 1995).

To achieve two-dimensional fine focusing, one solution is to ensure that the bent crystal maintains a continuous cylindrical shape over a wide area of the crystal. We designed a $\operatorname{Si}(111)$ rectangular slotted crystal with thick and wide stiffening wings, the so-called 'winged crystal', for use with a four-point crystal bender. The amount of anticlastic bending was analyzed by finite element analysis (FEA). We optimized the aspect ratio (length-to-width ratio) of the winged crystal to minimize anticlastic bending while maintaining a continuous cylindrical shape and parallelism to the first crystal within the Darwin width.

We report the focusing performance using the winged crystal. Preliminary results are also shown for the diffraction of a small protein crystal of hen-egg lysozyme with a high signal-to-noise ratio by matching the beam size to the sample size. 


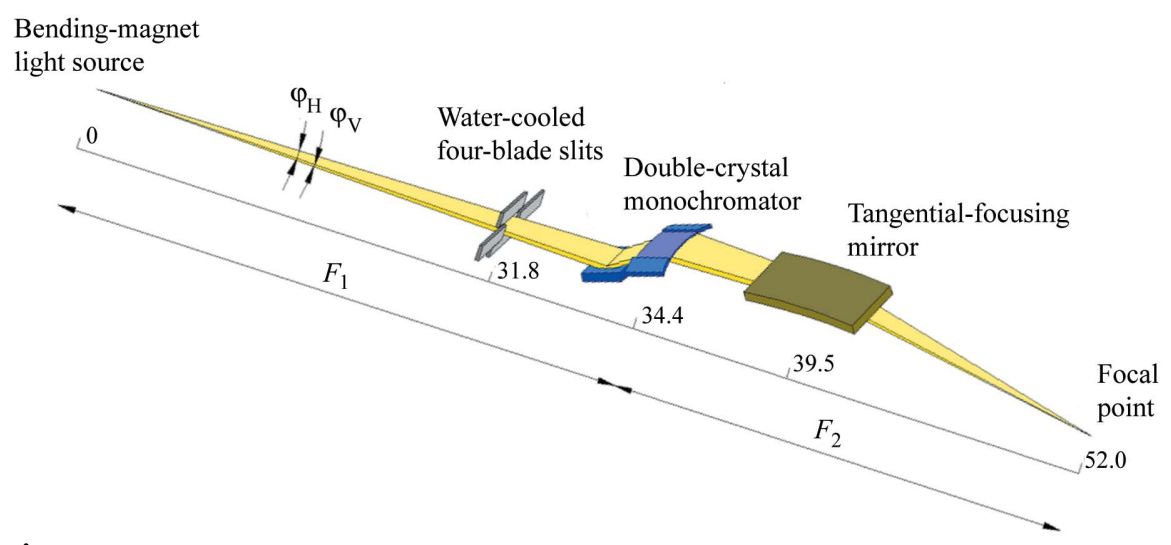

Figure 1

Schematic layout of BL26B1 and BL26B2. $\varphi_{\mathrm{H}}$ and $\varphi_{\mathrm{V}}$ are the horizontal and vertical acceptance angles of the incident X-rays from the bending-magnet light source, respectively. $F_{1}$ and $F_{2}$ are the distances from the double-crystal monochromator to the light source and focal point, respectively. The distances between components are presented in meters.

\section{Sagittal focusing crystal}

The characterization of two-dimensional focusing by a bent crystal with a tangential-focusing mirror was carried out using the optical geometry of beamlines BL26B1 and BL26B2 (RIKEN Structural Genomics beamlines I and II) of SPring-8 (Ueno et al., 2006). Fig. 1 shows a schematic layout of BL26B1 and BL26B2. The maximum acceptance angles of the doublecrystal monochromator, $\varphi_{\mathrm{H}}$ and $\varphi_{\mathrm{V}}$, are $1.5 \mathrm{mrad}$ and $200 \mu \mathrm{rad}$, respectively. Water-cooled four-blade slits and a SPring- 8 standard double-crystal monochromator (Uruga et al., 2001; Yabashi et al., 1999) are located at a distance of $31.8 \mathrm{~m}$ and $34.4 \mathrm{~m}$ from the light source, respectively. A directly watercooled $\mathrm{Si}(111)$ crystal (Nisawa et al., 2013) was used as the first crystal of the double-crystal monochromator. Figs. 2(a) and 2 (b) show schematic drawings of the four-point crystal bender for the second crystal (Kohzu Precision), which has a higher mechanical reliability on the bending mechanisms against the reactive force of a bent crystal. The rhodium-coated tangential-focusing mirror is located at a distance of $39.5 \mathrm{~m}$ from the light source. The glancing angle of the mirror $\theta_{\mathrm{m}}$ was set to $3.6 \mathrm{mrad}$. The focal point of the two-dimensional focusing $\mathrm{X}$-ray beam is $52.0 \mathrm{~m}$ from the light source. The magnification of sagittal focusing, $M\left(=F_{2} / F_{1}\right)$, is approximately $1 / 2$. By performing ray-tracing calculations for the ideal cylindrical shapes of the crystal and mirror, we found that the horizontal beam width is limited owing to aberration. Fig. 3 shows the results of the ray-tracing calculations using SHADOW (Welnak et al., 1994) running under XOP (X-ray Oriented Programs; Sanchez del Rio \& Dejus, 1997, 1998). The twodimensional focal beam images become a sharply defined core without a single-sided tail owing to the external curvature of the crystal (Sparks et al., 1980) when $\varphi_{\mathrm{H}}$ is fixed at $0.7 \mathrm{mrad}$, as shown in Figs. 3(a) and 3(b). The optimal horizontal acceptance angle is $0.7 \mathrm{mrad}$, which reduces the tails to less than $10 \mu \mathrm{m}$ for energies of 8 to $17.5 \mathrm{keV}$. The horizontal footprint of the X-ray beam on the second crystal surface is approximately $24 \mathrm{~mm}$ for an acceptance angle of $0.7 \mathrm{mrad}$. We have designed the rectangular slotted crystal based on the dimen- sions of a SPring- 8 standard sagittal crystal (Yoneda et al., 2003, 2005) taking into account the results of ray-tracing calculations.

We should carefully examine the aspect ratio of the sagittal crystal to avoid anticlastic bending effects. Fig. 4 shows a schematic of the anticlastic deformation in an isotropic rectangular thin crystal $(2 X \times 2 Y)$ with a simply supported edge boundary condition; a constant moment is applied along the edges $(y= \pm Y)$. The anticlastic curvature is defined by a function of the crystal's aspect ratio (length-to-width ratio), $\gamma=Y / X$. Kushnir et al. (1993) have shown that in order to obtain a small curvature at the crystal center point $(x=y=0)$ the aspect ratio must be large $(\gamma>7)$ or it must be equal to a certain golden value, $\gamma_{0}$. For a Poisson coefficient of 0.262 , the golden value is 2.360 . Under clamped edge boundary conditions $[z(x, y)=0$ and $\partial z / \partial x=$ 'constant' at $x= \pm X]$ the golden value decreases to 1.42 . However, it is difficult to provide a simply supported edge boundary condi-

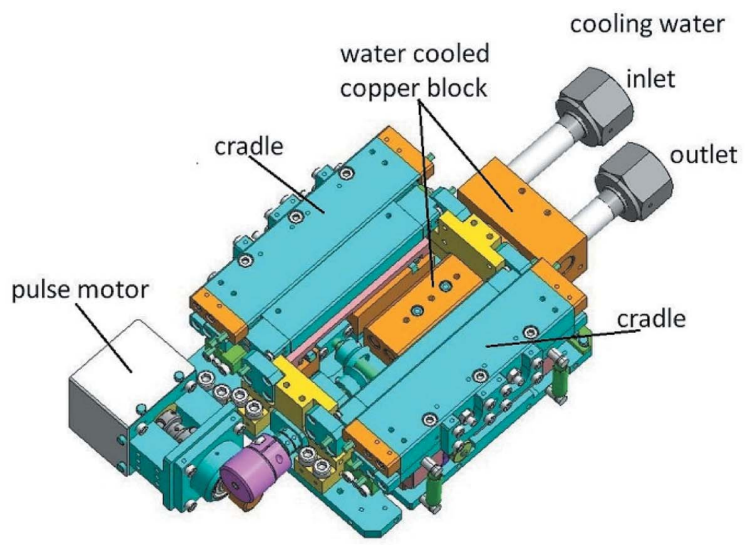

(a)

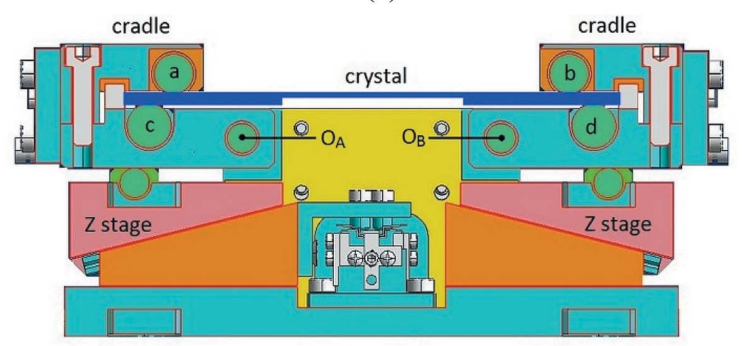

(b)

Figure 2

(a) Schematic of the four-point crystal bender for the second crystal (Kohzu Precision) and (b) the actual bending mechanism when the crystal is flattened. The geometrical condition of this bender is the same as that of a SPring-8 standard bender (Yoneda et al., 2001). The crystal was clamped with cylindrical rollers $(a, b, c$ and $d$ ) of the cradles. The effective length of the rollers is $110 \mathrm{~mm}$. The center distance of the upper cylindrical rollers $(a$ and $b)$ is $80 \mathrm{~mm}$, and that of the lower cylindrical rollers $(c$ and $d)$ is $90 \mathrm{~mm}$. The bending was performed by rotating the cradles around $O_{A}$ and $O_{B}$ owing to the vertical motion of mechanically linked $Z$ stages. 

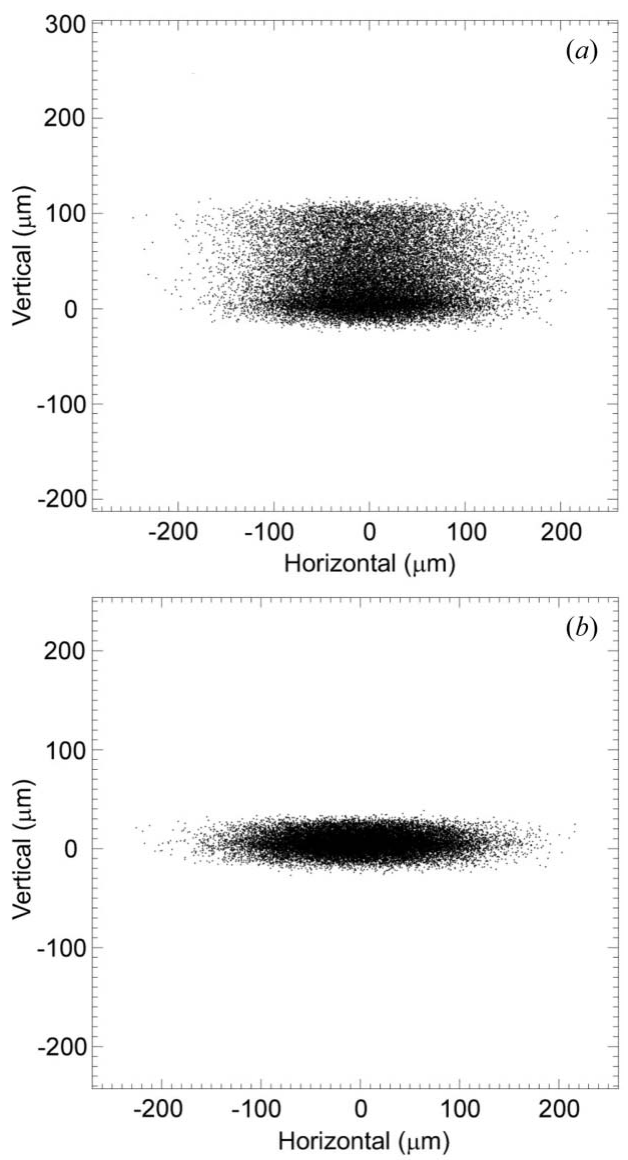

Figure 3

Ray-tracing results for focal beam images at $12.4 \mathrm{keV}$ in the ideal case using the geometry of BL26B1 and BL26B2 (Fig. 1) with horizontal acceptance angle $\varphi_{\mathrm{H}}$ values of $(a) 1.5 \mathrm{mrad}$ and $(b) 0.7 \mathrm{mrad}$. The vertical acceptance of the incident X-rays, $\varphi_{\mathrm{V}}$, was set to $30 \mu \mathrm{rad}$ in both cases. The light source is a $0.679 \mathrm{~T}$ bending-magnet source with source electron beam sizes of $\sigma_{x}=106 \mu \mathrm{m}$ and $\sigma_{y}=13 \mu \mathrm{m}$ and emittances of $\varepsilon_{x}=3.4 \times$ $10^{-9} \mathrm{~m} \mathrm{rad}$ and $\varepsilon_{y}=6.8 \times 10^{-12} \mathrm{~m}$ rad (Tanaka \& Kitamura, 2001). The sagittal crystal radius of curvature $R_{\mathrm{s}}$ is $3.71 \mathrm{~m}$. The glancing angle of the tangential-focusing mirror $\theta_{\mathrm{m}}$ is $3.6 \mathrm{mrad}$, and the radius of curvature $R_{\mathrm{m}}$ is $5.27 \mathrm{~km}$.

tion in a four-point bender (Quintana et al., 1995; Kushnir et al., 1993). We have determined an optimum design of the crystal by FEA using the ANSYS program (ANSYS, 2007) with the physically controllable clamped crystal boundary condition in a four-point crystal bender.

The final design of the winged crystal is shown in Fig. 5. The crystal has thick and wide wings, which improve its rigidity, similarly to the stiffening effect of ribs for a ribbed crystal. The dimensions of the crystal were fixed to values required for an advanced four-point crystal bender, as shown in Fig. 2. The aspect ratio of a SPring- 8 standard sagittal crystal (Yoneda et al., 2003, 2005) is $\gamma=1.435$, corresponding to a width $2 X$ of $62.7 \mathrm{~mm}$ in the thin region. The optimized width obtained by the analysis is $30 \mathrm{~mm}$ (Fig. 5), corresponding to a ratio of $\gamma=3$ and an acceptance angle of $\varphi_{\mathrm{H}}=0.9 \mathrm{mrad}$. This is larger than the optimum value for two-dimensional focusing of $\varphi_{\mathrm{H}}=$ $0.7 \mathrm{mrad}$.

Figs. $6(a)$ and $6(b)$ show the FEA results for the sagittal radius $\left(R_{\mathrm{s}}\right)$ distributions of the slotted area across the meri-

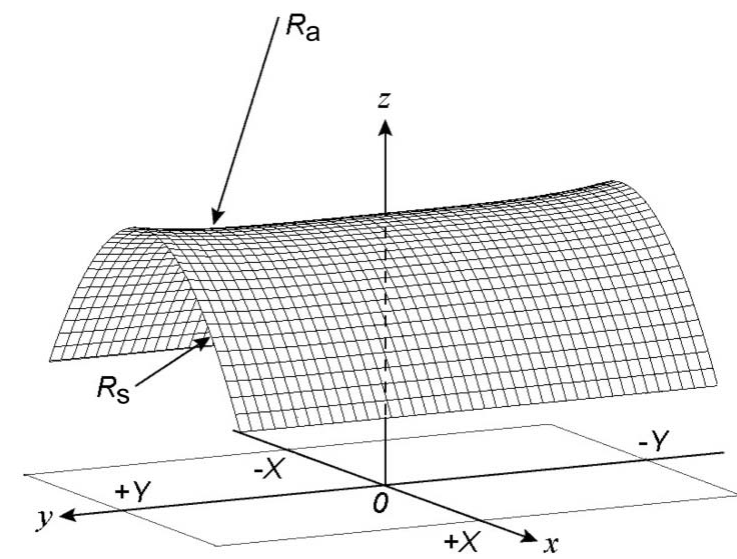

Figure 4

Typical shape of a bent crystal of dimensions $2 X \times 2 Y$ subjected to anticlastic bending. $R_{\mathrm{s}}$ is the sagittal radius and $R_{\mathrm{a}}$ is the anticlastic radius. The length-to-width ratio $(\gamma)$ is given by $Y / X$, where $X$ and $Y$ are the halfwidth and half-length of the crystal, respectively.

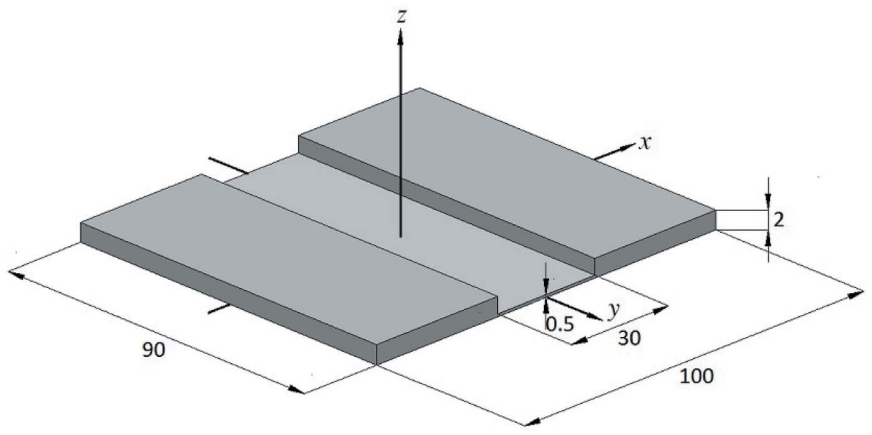

Figure 5

Schematic of the $\mathrm{Si}(111)$ rectangular slotted second crystal (winged crystal). The dimensions of the crystal are given in millimeters. The geometry of the slotted area corresponds to that shown in Fig. 4, i.e. $2 X=$ $30 \mathrm{~mm}, 2 Y=90 \mathrm{~mm}$ and $\gamma=3.0$.

dional center $(Y=0)$ and the slope error distributions along the meridional centerline owing to the anticlastic bending, respectively. FEA simulations were performed with clamped conditions in a four-point crystal bender (see Figs. 5 and 2) fixed at $y(x, z)=y( \pm 40,0)$, and with the applied uniform displacement $U Z$ at $y(x, z)=y( \pm 45,2)$. The Poisson coefficient for the $\mathrm{Si}(111)$ plane and the sagittal radius were set to 0.262 and $4.1 \mathrm{~m}$, respectively. The FEA results of the crystals were presented with aspect ratios of $1.435,2,3$ and 4 . The widths of $2 X$ for those aspect ratios were $62.7 \mathrm{~mm}, 45 \mathrm{~mm}$, $30 \mathrm{~mm}$ and $22.5 \mathrm{~mm}$, respectively. The applied uniform displacement $U Z$ values for the crystals were $-40 \mu \mathrm{m}$, $-30 \mu \mathrm{m},-20 \mu \mathrm{m}$ and $-17 \mu \mathrm{m}$, respectively. From these results it was clarified that the anticlastic bending effect decreases with increasing aspect ratio $\gamma$. The anticlastic bending is almost reduced over a slotted area with $\gamma=4$. This aspect ratio $\gamma$ is smaller than other reported large aspect ratios of 6 (Schulze et al., 1998), 6.6 (Bilsborrow et al., 2006) and 7.6 (Frenkel et al., 1996). In this case, $2 X$ of $22.5 \mathrm{~mm}$ is smaller than the $24 \mathrm{~mm}$ required for the optimal horizontal acceptance angle of $0.7 \mathrm{mrad}$ for two-dimensional focusing. When 

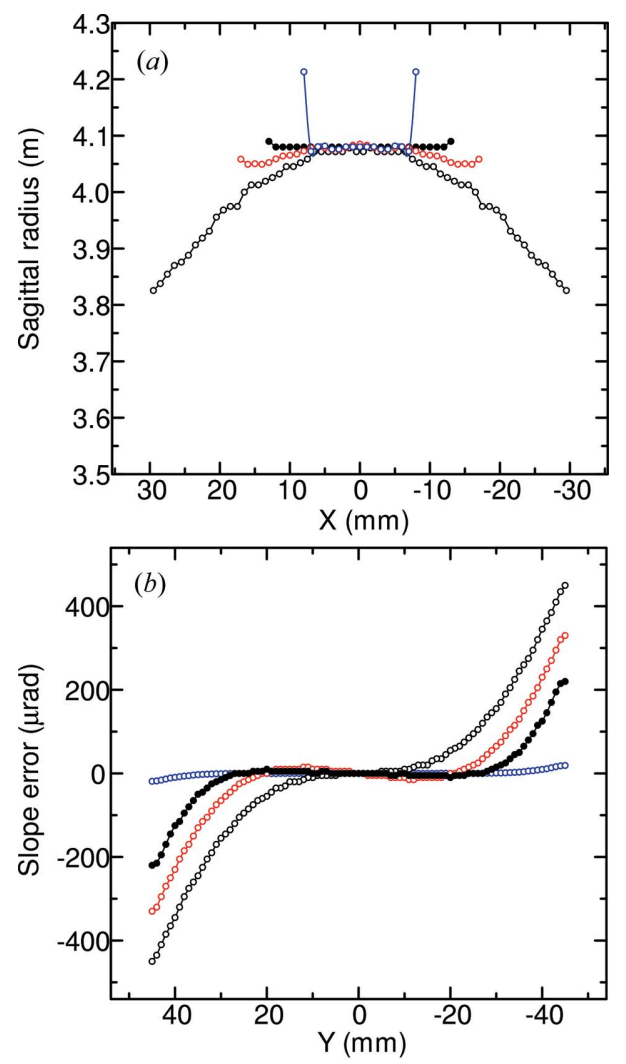

Figure 6

FEA results for crystals with $\gamma=1.435$ (black open circles), 2 (red open circles), 3 (black filled circles) and 4 (blue open circles); ( $a$ ) sagittal radius distributions along the sagittal $X$-direction for $Y=0$, and $(b)$ slope error distributions along the meridional $Y$-direction for $X=0$.

we set the aspect ratio $\gamma$ to $4,2 X$ should be $30 \mathrm{~mm}$ to ensure the same optimal horizontal acceptance angle of $0.7 \mathrm{mrad}$ as that of the $\gamma=3$ crystal, and the crystal length $2 Y$ should be stretched from $90 \mathrm{~mm}$ to $120 \mathrm{~mm}$. Since $2 Y$ of $120 \mathrm{~mm}$ is larger than the effective length of the rollers $(110 \mathrm{~mm})$ in our fourpoint bender, the stretched $\gamma=4$ crystal is impractical. In practice, the $\gamma=3$ crystal with $2 X=30 \mathrm{~mm}$ satisfies the requirements for the optimal horizontal acceptance angle of $0.7 \mathrm{mrad}$. For the winged crystal with $\gamma=3$, the sagittal radius distribution maintains a continuous cylindrical shape with minimal anticlastic bending over a wide central area of $26 \mathrm{~mm}$ $(2 X) \times 50 \mathrm{~mm}(2 Y)$. In this area the maximum slope error is $5 \mu \mathrm{rad}$, indicating that anticlastic bending is negligible. In the same area for the $\gamma=2$ and 1.435 crystals, the maximum slope errors are $20 \mu \mathrm{rad}$ and $90 \mu \mathrm{rad}$, respectively. In addition, the FEA results of the golden value of $1.42(2 X=63.38 \mathrm{~mm})$ were almost the same as those of 1.435 in our analysis. The aspect ratio $\gamma=3$ deviates from Kushnir's ideal aspect ratio, golden value or $\gamma>7$ to prevent anticlastic bending effects. The FEA results show that the anticlastic bending of the rectangular slotted crystal with aspect ratio $\gamma=3$ can be minimized under our clamping boundary condition. We have determined the aspect ratio $\gamma$ of the winged crystal to be 3 by ray-tracing calculation and FEA analysis to achieve two-dimensional fine focusing, as shown in Fig. 6 .

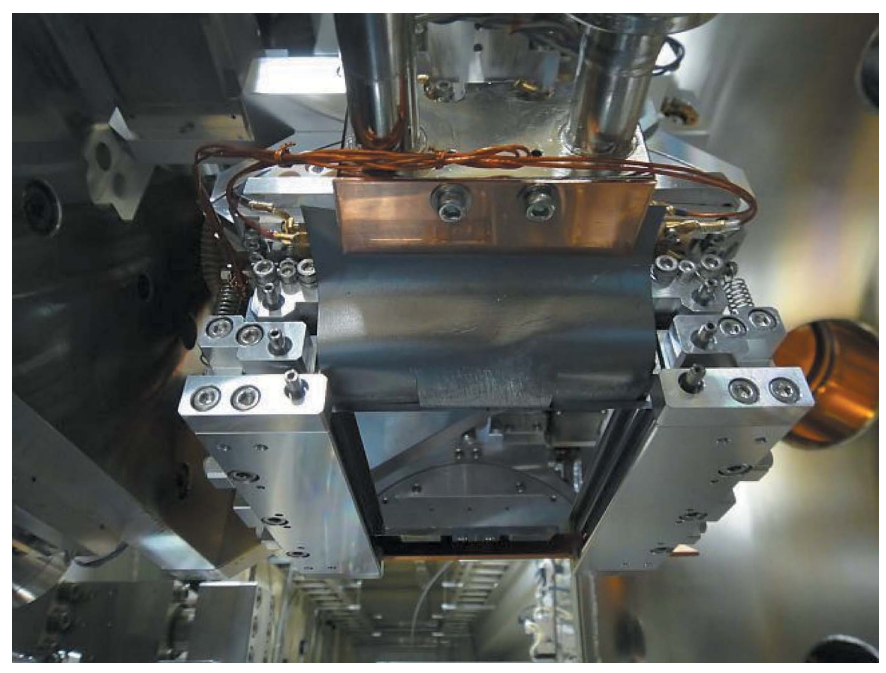

Figure 7

Four-point bender for the winged crystal installed in the double-crystal monochromator as a second crystal.

\section{Performance test at synchrotron radiation beamlines}

The Si(111) winged crystal with $\gamma=3$ (Fig. 5) was manufactured by high-precision machining and chemical etching (Sharan Instruments), and mounted on the second crystal stage with the advanced four-point crystal bender (Kohzu Precision). It was installed in the second crystal stage of the double-crystal monochromator at BL26B1 as shown in Fig. 7. The bender was cooled by water to prevent heat loading due to Compton scattered X-rays from the first crystal. The winged second crystal was also indirectly cooled by water via thin pyrolytic graphite sheets (PGS, Panasonic), which were brought into contact with the bottom surface of the crystal using a liquid gallium-indium (Ga-In) alloy. The horizontal and vertical acceptance angles of the incident $\mathrm{X}$-rays from the bending-magnet light source were set to $0.7 \mathrm{mrad}$ and $30 \mu \mathrm{rad}$, respectively, using the water-cooled four-blade slits.

The rocking curves were measured using an ion chamber to assess the effect of the winged crystal on cylindrical bending. The two-dimensional beam profiles at the focal point were observed using an X-ray beam monitor combined with a charge-coupled device (CCD) camera in the energy range from 8 to $17.5 \mathrm{keV}$, lower than the critical energy of a mirror reflection. The photon flux for several X-ray energies at the focal point was measured using a Si pin-photodiode detector.

\section{Results and discussion}

Fig. 8 shows the obtained rocking curves at $12.4 \mathrm{keV}$ for a bent crystal of $R_{\mathrm{s}}=4 \mathrm{~m}$ and for a flattened crystal of $R_{\mathrm{s}}=\infty$. $R_{\mathrm{s}}$ was determined from the amount of vertical motion of the mechanically linked $Z$ stages. The FWHM of the flattened crystal of $R_{\mathrm{s}}=\infty$ was $4 \mu \mathrm{rad}$ broader than the FWHM calculated from the double-crystal rocking curve. The beam sizes (vertical $\times$ horizontal) at the focal position for $R_{\mathrm{s}}=4 \mathrm{~m}$ and $R_{\mathrm{s}}=\infty$ were $1.8 \mathrm{~mm} \times 140 \mu \mathrm{m}$ and $1.5 \mathrm{~mm} \times 35 \mathrm{~mm}$, 


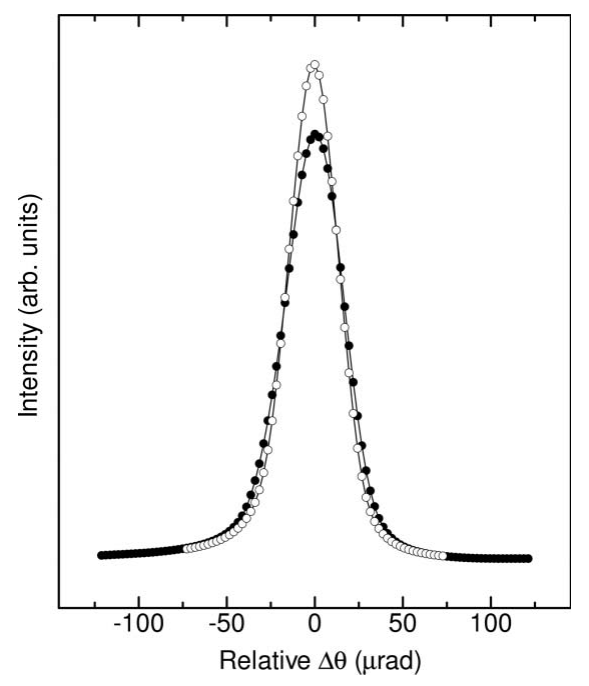

Figure 8

Observed rocking curves for total flux at $12.4 \mathrm{keV}$ using a fully sagittally focused crystal of $R_{\mathrm{s}}=4 \mathrm{~m}$ (filled circles) and a flattened crystal of $R_{\mathrm{s}}=\infty$ (open circles). The full widths at half-maximums (FWHMs) of the rocking curves for the fully focused and flattened crystals are 39 and $34 \mu \mathrm{rad}$, respectively. The FWHM of the double-crystal rocking curve calculated by using $X O P$ is $30 \mu \mathrm{rad}$.

respectively. The peak intensity (total photon flux) was approximately $8 \%$ lower and the rocking curve was approximately $5 \mu \mathrm{rad}$ broader for the bent crystal of $R_{\mathrm{s}}=4 \mathrm{~m}$. The flux density at the sample position with focusing increased approximately 200 times more than that for the flattened crystal of $R_{\mathrm{s}}=\infty$. Significant reductions in peak flux and energy resolution due to bending were not observed. The symmetrical rocking-curve profile suggests that the winged crystal was bent cylindrically and that anticlastic bending was minimized.

The spatial profiles obtained by two-dimensional focusing with a tangential-focusing mirror were observed in the energy range from 8 to $17.5 \mathrm{keV}$. Figs. $9(a)-9(c)$ show the calculated two-dimensional focusing beam images obtained by raytracing calculations using SHADOW software for energies of $8,12.4$ and $17.5 \mathrm{keV}$, respectively, where $\left(a^{\prime}\right)-\left(c^{\prime}\right)$ are the corresponding observed spatial profiles at the focal point. The observed spatial profiles have a sharply defined core for each energy. These profiles agree well with the results of the raytracing calculations. The dimensions of the focused beams are compared with those in the ray-tracing results in Table 1. The observed vertical widths of the focusing beams are larger than those in the ray-tracing results. This broadening of vertical widths may be caused by the undesirable slope errors of the first crystal, winged crystal and tangentially bent cylindrical mirror. The slope error of the tangentially bent cylindrical mirror is due to a surface figure error from the ideal cylindrical shape. The slope error of the winged crystal is due to the mounting stress of rollers and the thickness variation of the crystal (Schulze et al., 1998). At higher energies the thickness variation and anticlastic bending effects of the crystal increase with the decrease in bending radius, and the footprint in the meridional direction on the winged crystal surface becomes large. The effective slope error of the winged crystal becomes
Table 1

Comparison between two-dimensional focusing dimensions obtained by ray-tracing and observation for energies of $8,12.4$ and $17.5 \mathrm{keV}$.

$V_{\text {cal }}$ and $H_{\text {cal }}$ are the vertical and horizontal dimensions (FWHM), respectively, of focal images obtained by ray-tracing calculations using $S H A D O W$ software. $V_{\text {obs }}$ and $H_{\text {obs }}$ are the observed values.

\begin{tabular}{llllll}
\hline $\begin{array}{l}\text { Energy } \\
(\mathrm{keV})\end{array}$ & $R_{\mathrm{s}}(\mathrm{m})$ & $V_{\text {cal }}(\mu \mathrm{m})$ & $H_{\text {cal }}(\mu \mathrm{m})$ & $V_{\text {obs }}(\mu \mathrm{m})$ & $H_{\text {obs }}(\mu \mathrm{m})$ \\
\hline 8 & 6.2 & 18.6 & 132.4 & 44.5 & 139.5 \\
12.4 & 4.0 & 22.5 & 133.6 & 40.1 & 139.4 \\
17.5 & 2.8 & 24.0 & 132.9 & 35.0 & 142.2 \\
\hline
\end{tabular}

slightly larger at higher energies. This slope error reduces the vertical divergence of the output X-ray beam from the doublecrystal monochromator, and the observed vertical width of the focusing beam decreases at higher energies.

Table 2 shows the observed photon fluxes, the ratios of the photon fluxes and the ratios of the focal beam cross sections compared with those for the crystal with $\gamma=1.435$, which were observed at BL26B2 with the same acceptance angles of $\varphi_{\mathrm{H}}=$ $0.7 \mathrm{mrad}$ and $\varphi_{\mathrm{V}}=30 \mu \mathrm{rad}$. It is clear that the improvement factors of the photon flux and the cross section for the winged
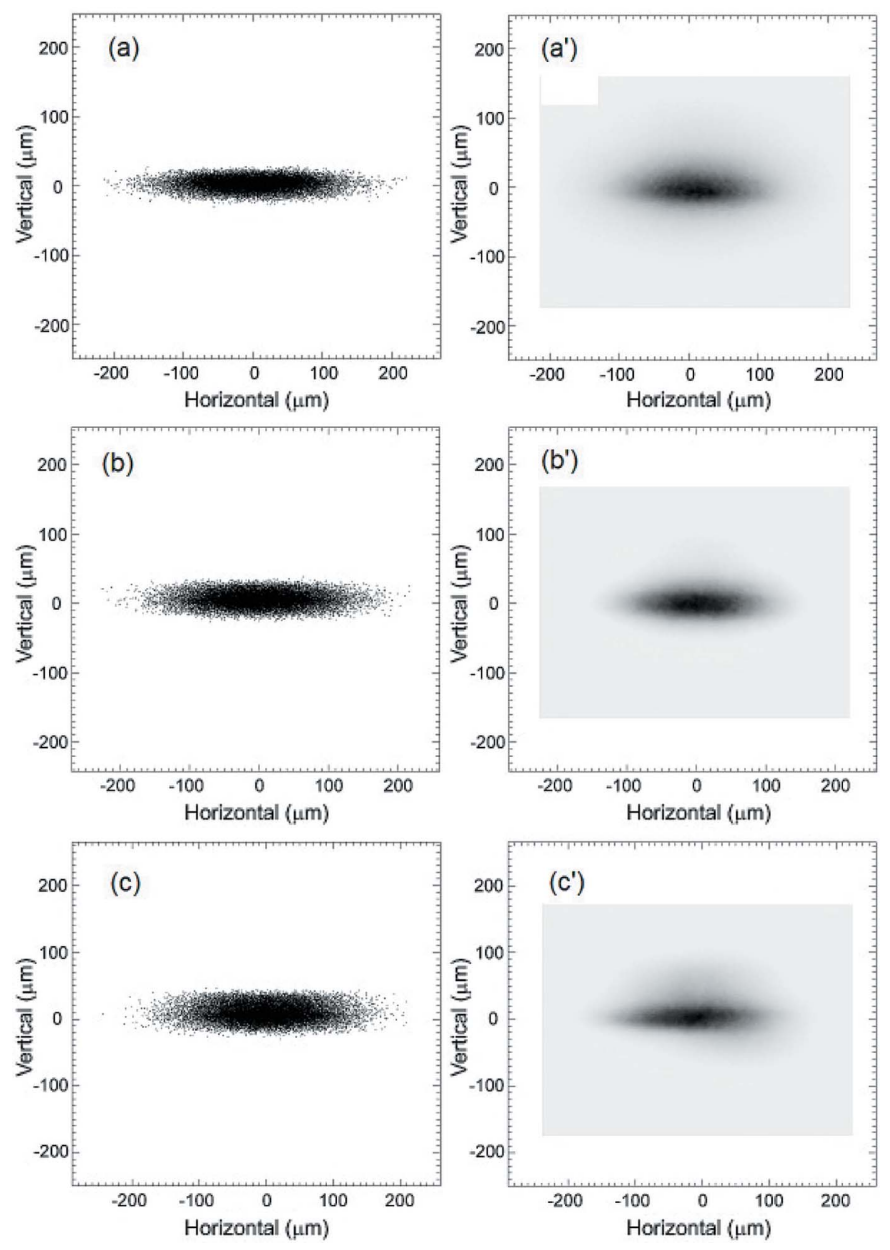

Figure 9

Spatial profiles obtained by two-dimensional focusing. (a)-(c) Calculated beam images obtained by ray-tracing calculations using SHADOW software for energies of $8,12.4$ and $17.5 \mathrm{keV}$, respectively. $\left(a^{\prime}\right)-\left(c^{\prime}\right)$ Observed beam profiles at the focal point. 
Table 2

Observed photon flux for two-dimensional focusing with energies of 8, 12.4 and $17.5 \mathrm{keV}$.

$I_{\gamma=3}$ and $I_{\gamma=1.435}$ indicate the observed photon fluxes for crystals with $\gamma=3$ and 1.435 , respectively. $S_{\gamma=3}$ and $S_{\gamma=1.435}$ indicate the observed focal beam cross sections for crystals with $\gamma=3$ and 1.435, respectively. $D_{\gamma=3}$ and $D_{\gamma=1.435}$ indicate the observed flux densities for crystals with $\gamma=3$ and 1.435 , respectively.

\begin{tabular}{|c|c|c|c|c|}
\hline $\begin{array}{l}\text { Energy } \\
(\mathrm{keV})\end{array}$ & $\begin{array}{l}\text { Photon flux } \\
\left(\text { photons s }^{-1}\right)\end{array}$ & $\begin{array}{l}\text { Flux ratio } \\
\left(I_{\gamma=3} / I_{\gamma=1.435}\right)\end{array}$ & $\begin{array}{l}\text { Cross-section ratio } \\
\left(S_{\gamma=3} / S_{\gamma=1.435}\right)\end{array}$ & $\begin{array}{l}\text { Flux density ratio } \\
\left(D_{\gamma=3} / D_{\gamma=1.435}\right) \\
\end{array}$ \\
\hline 8 & $4.56 \times 10^{10}$ & 1.1 & 0.63 & 1.75 \\
\hline 12.4 & $1.22 \times 10^{11}$ & 1.1 & 0.71 & 1.55 \\
\hline 17.5 & $9.40 \times 10^{10}$ & 1.5 & 0.53 & 2.83 \\
\hline
\end{tabular}

map for identifying each amino acid residue in the main chain was obtained.

A comparative measurement was conducted to investigate the improved performance of the monochromator to enhance the weak signals of high-resolution diffraction spots. Two sets of diffraction data at the same exposure time and oscillation width from a lysozyme crystal were obtained with the sagittal and conventional focusing beams. The conventional focusing beam was provided

crystal at the focal position are significantly larger at higher energies, where the sagittal radius is smaller and the footprint in the meridional direction is larger. The higher flux density ratios for the winged crystal are mainly caused by the decrease in beam size at the focal position. A key to achieving a small vertical beam size in the system is to eliminate the vertical smearing that can be seen in Fig. 3. Since the anticlastic bending of the winged crystal has been minimized for a wide sagittal radius $R_{\mathrm{s}}$, as shown in Fig. 6, two-dimensional fine focusing with a tangential-focusing mirror was successfully achieved with a very small loss of photon flux.

The two-dimensional finely focused beam with high photon flux enables the observation of X-ray diffraction data with a high signal-to-noise ratio from small protein crystals, because the diffraction signal is enhanced and the background scattering around the sample is reduced. Fig. 10 shows an example of an observed diffraction image of a protein crystal sample (hen-egg lysozyme) obtained by the two-dimensional focusing of an X-ray beam with a photon flux of $\sim 10^{11}$ photons s $^{-1}$ and a photon energy of $12.4 \mathrm{keV}(\lambda=1 \AA)$. The crystal size was $70 \mu \mathrm{m} \times 100 \mu \mathrm{m} \times 80 \mu \mathrm{m}$, and the oscillation condition was a $1^{\circ}$ rotation with $2 \mathrm{~s}$ exposure per frame. The diffraction spots were very clear, and no distortion was observed up to the highest resolution of the detector aperture. The statistics obtained by data processing using the $H K L-2000$ program package (Otwinowski \& Minor, 1997) are shown in Table 3. As a result of the structure analysis by deriving structure factors from the data set shown in Table 3, a clear electron density

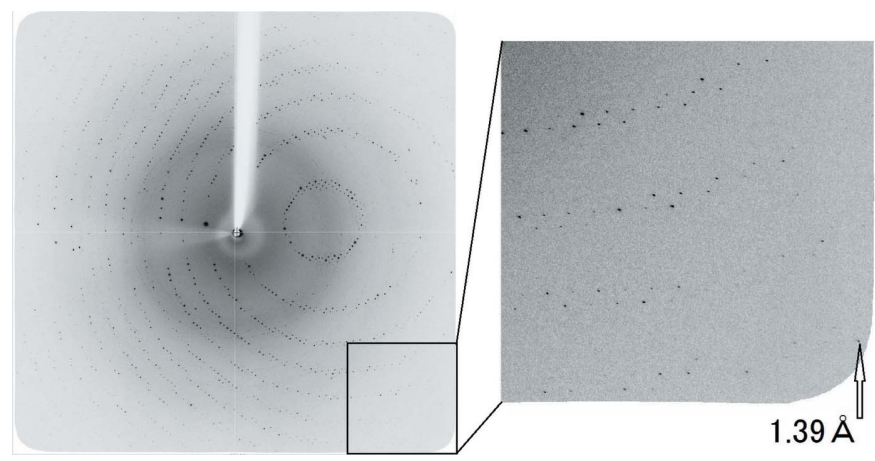

Figure 10

Diffraction image of a protein crystal (hen-egg lysozyme) observed using a CCD area detector (Saturn A200, Rigaku) located $150 \mathrm{~mm}$ from the sample. The inset shows the corresponding resolution of the diffraction spot. by combining plane-plane crystals and a tangentially bent cylindrical mirror. A comparison of the statistics is shown in Table 4 . The data set obtained with the sagittal focusing beam showed significantly improved $R$-merge and Mean $I / \sigma(I)$, particularly in high-resolution shells, owing to the enhanced signal with the sagittally focusing beam.

\section{Summary}

We designed a Si(111) winged crystal to minimize anticlastic bending in sagittal focusing. The crystal had a thin rectangular area at its center, an aspect ratio $(\gamma)$ of 3 , and wide stiffening

Table 3

Statistics of the diffraction data set for a hen-egg lysozyme crystal.

Data collection conditions: oscillation range: $0-360^{\circ}$ in $1^{\circ}$ steps (360 images); exposure time: $2 \mathrm{~s}$ per frame. Values in parentheses refer to the highestresolution shell (1.75-1.69 $\AA$ ). $R$-merge is the residual among equivalent reflections expressed as $\Sigma|I-\langle I\rangle| / \Sigma\langle I\rangle$.

\begin{tabular}{ll}
\hline Space group & $P 4_{3} 2_{1} 2$ \\
Cell constants $(\AA)$ & $a=78.98, b=36.98$ \\
Wavelength $(\AA)$ & 1.00 \\
Resolution $(\AA)$ & $50.0-1.69(1.75-1.69)$ \\
No. of observations & 359121 \\
Unique reflections & 13650 \\
Data completeness $(\%)$ & $98.9(91.4)$ \\
Mean $I / \sigma(I)$ & $121.9(61.0)$ \\
$R$-merge & $0.044(0.081)$ \\
\hline
\end{tabular}

\section{Table 4}

Statistics of comparative diffraction data set for a hen-egg lysozyme crystal.

Two data sets are obtained from an identical sample in different beam set-ups of sagittal focusing and conventional optics. The crystal size is $100 \mu \mathrm{m} \times 90 \mu \mathrm{m}$ $\times 60 \mu \mathrm{m}$. Data collection conditions: oscillation range: 0 to $180^{\circ}$ in $1^{\circ}$ steps (180 images); exposure time: $3 \mathrm{~s}$ per frame. Values in parentheses refer to the highest-resolution shell $(1.76-1.70 \AA)$. $R$-merge is the residual among equivalent reflections expressed as $\Sigma|I-\langle I\rangle| / \Sigma\langle I\rangle$.

\begin{tabular}{lll}
\hline & Optics setting & \\
\cline { 2 - 3 } & Sagittal focusing & Conventional \\
\hline Space group & $P 4_{3} 2_{1} 2$ & $P 4_{3}{ }_{1} 2$ \\
Cell constants $(\AA)$ & $a=78.77, b=36.92$ & $a=78.87, b=36.97$ \\
Wavelength $(\AA)$ & 1.00 & 1.00 \\
Resolution $(\AA)$ & $50.0-1.70(1.76-1.70)$ & $50.0-1.70(1.76-1.70)$ \\
No. of observations & 176411 & 176133 \\
Unique reflections & 13356 & 13382 \\
Data completeness $(\%)$ & $99.7(99.9)$ & $99.9(100.0)$ \\
Mean $I / \sigma(I)$ & $84.7(34.4)$ & $66.0(15.1)$ \\
$R$-merge & $0.036(0.090)$ & $0.044(0.198)$ \\
\hline
\end{tabular}


wings. It also had a uniform sagittal radius and reduced surface slope error due to anticlastic bending compared with a crystal of $\gamma=1.435$. Two-dimensional focusing was achieved with a $\mathrm{Si}(111)$ winged crystal and a tangential-focusing mirror without any undesirable aberrations in the energy range from 8 to $17.5 \mathrm{keV}$. This focusing system can be used to obtain highquality data with a high signal-to-noise ratio for the diffraction of small protein crystals. Achievements of structural biology research have already been published (e.g. Kounosu et al., 2008; Saino et al., 2011). The winged crystal proposed here is also applicable to sagittal focusing with a $\mathrm{Si}(311)$ crystal monochromator in an adjustable inclined geometry (Uruga $e t$ al., 2001), and performance tests are ongoing.

We would like to thank Drs K. Takeshita, Y. Furukawa, H. Yamazaki, K. Sugimoto, T. Mochizuki and H. Ohashi for their useful discussions and support. We would also like to thank Kohzu Precision for manufacturing the crystal bender and Sharan Instruments Corporation for manufacturing the sagittal crystals. Financial support by Rigaku Corporation is gratefully acknowledged.

\section{References}

ANSYS (2007). ANSYS Release 11.0. ANSYS Inc., Canonsburg, PA, USA.

Bilsborrow, R. L., Atkinson, P. A., Bliss, N., Dent, A. J., Dobson, B. R. \& Stephenson, P. C. (2006). J. Synchrotron Rad. 13, 54-58.

Borsboom, M., Bras, W., Cerjak, I., Detollenaere, D., Glastra van Loon, D., Goedtkindt, P., Konijnenburg, M., Lassing, P., Levine, Y. K., Munneke, B., Oversluizen, M., van Tol, R. \& Vlieg, E. (1998). J. Synchrotron Rad. 5, 518-520.

Feng, L., Kang, L., Li, Z., Zhao, F. \& Xu, C. (2008). J. Synchrotron Rad. 15, 140-143.

Frenkel, A., Barg, B., Heald, S., Kim, K. H., Brown, F. \& Stern, E. A. (1996). Rev. Sci. Instrum. 67, 1-4.
Koshelev, I., Huang, R., Graber, T., Meron, M., Muir, J. L., Lavender, W., Battaile, K., Mulichak, A. M. \& Keefe, L. J. (2009). J. Synchrotron Rad. 16, 647-657.

Kounosu, A., Iwasaki, T., Baba, S., Hayashi-Iwasaki, Y., Oshima, T. \& Kumasaka, T. (2008). Acta Cryst. F64, 1146-1148.

Kushnir, V. I., Quintana, J. P. \& Georgopoulos, P. (1993). Nucl. Instrum. Methods Phys. Res. A, 328, 588-591.

Nisawa, A. et al. (2013). In preparation.

Nomura, M. \& Koyama, A. (1999). J. Synchrotron Rad. 6, 182-184.

Otwinowski, Z. \& Minor, W. (1997). Methods Enzymol. 276, 307-326.

Quintana, V. I., Kushnir, J. P. \& Rosenbaum, G. (1995). Nucl. Instrum. Methods Phys. Res. A, 362, 592-594.

Saino, H., Ukita, Y., Ago, H., Irikura, D., Nisawa, A., Ueno, G., Yamamoto, M., Kanaoka, Y., Lam, B. K., Austen, K. F. \& Miyano, M. (2011). J. Biol. Chem. 286, 16392-16401.

Sanchez del Rio, M. \& Dejus, R. J. (1997). Proc. SPIE, 3152, 148-157.

Sanchez del Rio, M. \& Dejus, R. J. (1998). Proc. SPIE, 3448, 340-345.

Schulze, C., Heidenreich, G., Auderset, H., Vermeulen, D. \& Freund, A. K. (1998). Proc. SPIE, 3448, 156-165.

Sparks, C. J. Jr, Borie, B. S. \& Hastings, J. B. (1980). Nucl. Instrum. Methods, 172, 237-242.

Sparks, C. J. Jr, Borie, B. S. \& Hastings, J. B. (1982). Nucl. Instrum. Methods, 195, 73-78.

Tanaka, T. \& Kitamura, H. (2001). J. Synchrotron Rad. 8, 1221-1228.

Ueno, G., Kanda, H., Hirose, R., Ida, K., Kumasaka, T. \& Yamamoto, M. (2006). J. Struct. Funct. Genom. 7, 15-22.

Uruga, T., Tanida, H., Yoneda, Y., Takeshita, K., Goto, S. \& Ishikawa, T. (2001). Nucl. Instrum. Methods Phys. Res. A, 467-468, 782784.

Welnak, C., Chen, G. J. \& Cerrina, F. (1994). Nucl. Instrum. Methods Phys. Res. A, 347, 344-347.

Yabashi, M., Yamazaki, H., Tamasaku, K., Goto, S., Takeshita, K., Mochizuki, T., Yoneda, Y. \& Ishikawa, T. (1999). Proc. SPIE, 3773, 2-13.

Yoneda, Y., Matsumoto, N., Furukawa, Y. \& Ishikawa, T. (2001). J. Synchrotron Rad. 8, 18-21.

Yoneda, Y., Matsumoto, N., Furukawa, Y. \& Ishikawa, T. (2003). AIP Conf. Proc. 705, 720-723.

Yoneda, Y., Matsumoto, N., Furukawa, Y. \& Ishikawa, T. (2005). Phys. Scr. T115, 995-997. 